\title{
Consequences of Zmat3 loss in c-MYC- and mutant KRAS-driven tumorigenesis
}

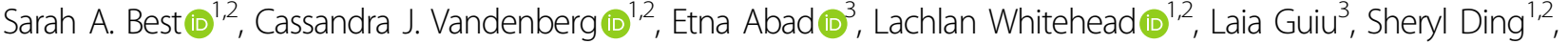

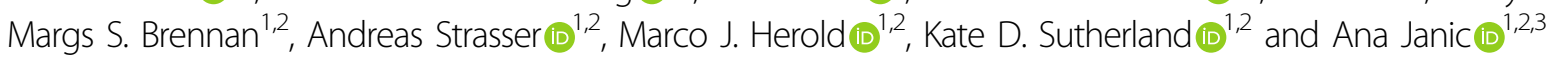

\begin{abstract}
TP53 is a critical tumor suppressor that is mutated in approximately 50\% of human cancers. Unveiling the downstream target genes of TP53 that fulfill its tumor suppressor function is an area of intense investigation. Zmat3 (also known as Wig-1 or PAG608) is one such downstream target of p53, whose loss in hemopoietic stem cells lacking the apoptosis and cell cycle regulators, Puma and p21, respectively, promotes the development of leukemia. The function of Zmat3 in tumorigenesis however remains unclear. Here, to investigate which oncogenic drivers co-operate with Zmat3 loss to promote neoplastic transformation, we utilized Zmat3 knockout mice in models of c-MYC-driven lymphomagenesis and $\mathrm{Kras}^{\mathrm{G}}{ }^{2 \mathrm{D}}$-driven lung adenocarcinoma development. Interestingly, unlike loss of $p 53$, Zmat3 germline loss had little impact on the rate of tumor development or severity of malignant disease upon either the $c-M Y C$ or Kras ${ }^{G 12 D}$ oncogenic activation. Furthermore, loss of Zmat3 failed to rescue Kras ${ }^{G 12 D}$ primary lung tumor cells from oncogeneinduced senescence. Taken together, we conclude that in the context of c-MYC-driven lymphomagenesis or mutant Kras ${ }^{G 12 D}$-driven lung adenocarcinoma development, additional co-occurring mutations are required to resolve Zmat3 tumor suppressive activity.
\end{abstract}

\section{Introduction}

P53 is a pivotal suppressor of cancer development and progression. The importance of p53 tumor suppression function and response to anti-cancer agents has driven the development of novel strategies to target the p53 pathway in cancer therapy ${ }^{1}$. Although the role of $\mathrm{p} 53$ in preventing tumor development has provoked intense investigation, the underlying mechanisms crucial in tumor suppression downstream of $\mathrm{p} 53$ have remained elusive ${ }^{2-4}$. Cell cycle arrest, cell senescence and apoptosis have been implicated in p53-mediated tumor suppression ${ }^{2,5,6}$. However, no spontaneous tumors arise in mice lacking Puma, Noxa, and

\footnotetext{
Correspondence: Kate D. Sutherland (sutherland.k@wehi.edu.au) or Ana Janic (ana.janic@upf.edu)

${ }^{1}$ The Walter and Eliza Hall Institute of Medical Research, 1G Royal Parade, Parkville, Melbourne, VIC 3052, Australia

${ }^{2}$ Department of Medical Biology, University of Melbourne, Parkville, Melbourne, VIC 3052, Australia

Full list of author information is available at the end of the article These authors share senior authorship: Kate D. Sutherland, Ana Janic Edited by U. Moll
}

$p 21$, the critical mediators of p53-induced apoptosis and G1/S cell cycle arrest ${ }^{7}$. Likewise, in several mouse tumor models in different cellular compartments, it has been demonstrated that p53 mutants defective in the induction of apoptosis, cell cycle arrest and cell senescence retain tumor-suppressor activity $^{8-10}$. Collectively, these studies demonstrate that p53 must suppress tumor development through currently underappreciated processes that act apart from, or in addition to, the induction of apoptosis, cell cycle arrest and cell senescence ${ }^{7,11-13}$.

Utilizing an in vivo shRNA screening approach, we recently demonstrated that Zmat3 acts as a tumor suppressor gene downstream of $\mathrm{p} 53$, with its loss cooperating with inactivation of cell cycle arrest, cell senescence and apoptosis inducers (PUMA and p21) in the development of hematopoietic malignancy ${ }^{14}$. Zmat3 has been described as an RNA-binding zinc-finger protein that is involved in posttranscriptional regulation of gene expression and as such is found expressed in a broad range of tissues ${ }^{15}$. Moreover, akin to being a p53 target gene, Zmat3 has been reported to

\section{(c) The Author(s) 2020}

(c) (i) Open Access This article is licensed under a Creative Commons Attribution 4.0 International License, which permits use, sharing, adaptation, distribution and reproduction in any medium or format, as long as you give appropriate credit to the original author(s) and the source, provide a link to the Creative Commons license, and indicate if changes were made. The images or other third party material in this article are included in the article's Creative Commons license, unless indicated otherwise in a credit line to the material. If material is not included in the article's Creative Commons license and your intended use is not permitted by statutory regulation or exceeds the permitted use, you will need to obtain permission directly from the copyright holder. To view a copy of this license, visit http://creativecommons.org/licenses/by/4.0/. 
exert bona fide roles in the control of cell proliferation and cell survival ${ }^{16}$, where it acts through the regulation of $p 53$ and $p 21 \mathrm{mRNA}^{17-20}$. Interestingly, while both decreased and increased Zmat 3 mRNA expression have been reported in human tumors ${ }^{15}$ the exact role of Zmat3 in tumorigenesis remains elusive. We therefore hypothesized that the function of Zmat3 in tumorigenesis may be context dependent, influenced by genetic background, cell type or oncogenic drivers specific to each tumor. To further investigate the functions of Zmat3 in tumor suppression, we evaluated the impact of its loss in c-MYC-driven lymphomagenesis and mutant $\mathrm{Kras}^{\mathrm{G} 12 \mathrm{D}}$-driven non-small cell lung cancer (NSCLC) $)^{21}$, in which the p53 pathway has been shown to play a critical tumor suppressive role ${ }^{22-24}$.

\section{Materials and methods Mice}

Animal experiments were conducted according to the regulatory standards approved by the Animal Ethics Committees of the Walter and Eliza Hall Institute of Medical Research (WEHI) and the Barcelona Biomedical Research Park (PRBB). E $\mu-M y c^{24}, p 53^{25,26}$, Kras $^{\text {LSL-G12D/+ }}$ mice ${ }^{21}$ and $Z$ mat ${ }^{14}$ mice have been previously described. All animals were maintained on a C57BL/6 background, and equal proportions of males and females were used in all experiments. Seven- to eight- week-old $\mathrm{Kras}^{\mathrm{LSL}-\mathrm{G} 12 \mathrm{D} /+}$ compound mice were intranasally (i.n.) infected with $20 \mu \mathrm{L}$ of $1 \times 10^{10} \mathrm{PFU} / \mathrm{mL}$ Ad5-CMV-Cre virus (University of Iowa Gene Transfer Core Facility) according to standard procedures ${ }^{27}$. Lungs were harvested at defined time points (6-, 10- and 16-weeks post i.n. infection) or when mice showed signs of morbidity for Kaplan-Meier survival analysis.

\section{qRT-PCR analysis}

Pre-B cells were treated with $5 \mathrm{~Gy} \gamma$-irradiation or with $10 \mu \mathrm{M}$ Nutlin3a (Nut3a), in the presence of $25 \mu \mathrm{M}$ QVDOPH (Sigma-Aldrich, St. Louis, MO, USA) in culture. WT and $Z$ mat $3^{-1-}$ mice, 2-5 months old, were either exposed to $8 \mathrm{~Gy} \gamma$-irradiation or left untreated and lung tissue was harvested after $6 \mathrm{~h}$. RNA was extracted using Trizol reagent (Invitrogen, Carlsbad, CA, USA) and converted to cDNA using the SuperScript First Strand Synthesis Kit (Thermo Fisher Scientific). Quantitative RT-PCR was performed using TaqMan probes (Thermo Fisher Scientific) for Zmat3 (Mm01292424_m1), Puma (Mm00519268_m1), p21 (Mm00432448_m1) and analyzed by the $\Delta \Delta \mathrm{CT}$ method relative to HMBS (Mm01143545_m1).

\section{Flow cytometry}

B lymphoid cells were isolated from bone marrow collected from both femurs and tibias of 4 week-old $E \mu-M y c$ mice and stained with the following antibodies: B220-PE (clone RA3-6B2), IgM-FITC (clone 5.1) and IgD-FITC (clone 11-26C). Viable pre-B cells (B220 $\left.{ }^{+} \operatorname{IgM}^{-} \operatorname{IgD}^{-} \mathrm{PI}^{-}\right)$were isolated by flow cytometry using an ARIA flow cytometer (Becton Dickinson, San Jose, CA, USA). $1 \times 10^{6}$ cells $/ \mathrm{mL}$ were cultured in DMEM (Thermo Fisher Scientific, Waltham, MA, USA), 10\% FBS (Sigma-Aldrich), $100 \mathrm{U} / \mathrm{mL}$ penicillin (Thermo Fisher Scientific), $100 \mu \mathrm{g} / \mathrm{mL}$ streptomycin (Sigma-Aldrich), $50 \mu \mathrm{M}$ 2-Mercaptoethanol and $100 \mu \mathrm{M}$ asparagine (Thermo Fisher Scientific) during the time-course. Antibodies were produced in-house.

Immunophenotyping of lymphomas was performed on single-cell suspensions stained with surface-markerspecific antibodies: CD43-BV650 (BD Biosciences; \#740464; dilution 1:800); B220-APC-Cy7 (BD Biosciences; \#552094; dilution 1:200); IgD-BV510 (BD Biosciences; \#563110; dilution 1:200); IgM-FITC (BD Biosciences; \#553437; dilution 1:100) to define pro/pre B cells $\left(\mathrm{B} 220^{+} \mathrm{CD} 43^{+}\right)$and Ig+ B cells (IgM and IgD). Samples were run on the LSR II (BD Biosciences). Dead cells $\left(\mathrm{PI}^{+}\right)$ were excluded from analysis.

For immune cell analysis of lung tissue, the left lobe of each lung was weighed and harvested in PBS for digestion to generate a single cell suspension and blocked as described previously ${ }^{28}$. Blocked cells were incubated with fluorochrome-conjugated antibodies to define key immune cell populations from the lymphoid and myeloid compartments: $\mathrm{B}$ cells $\left(\mathrm{CD} 45^{+} \mathrm{CD} 3^{-} \mathrm{CD} 19^{+}\right)$, $\mathrm{T}$ cells $\left(\mathrm{CD} 45^{+} \mathrm{CD}^{+}\right)$, NK cells $\left(\mathrm{CD} 45^{+} \mathrm{CD} 3^{-} \mathrm{NKp} 46^{+} \mathrm{DX} 5^{+}\right)$, alveolar macrophages $\left(\mathrm{CD} 45^{+} \mathrm{CD} 11 \mathrm{c}^{+} \mathrm{CD} 103^{-}\right), \mathrm{CD} 103$ dendritic cells $\left(\mathrm{CD} 45^{+} \mathrm{CD} 11 \mathrm{c}^{+} \mathrm{CD} 103^{+}\right)$, monocytes

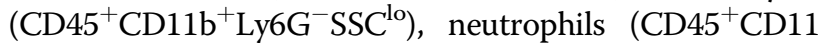
$\mathrm{b}^{+} \mathrm{Ly}_{6 \mathrm{G}^{+}}$) and eosinophils $\left(\mathrm{CD} 45^{+} \mathrm{CD} 11 \mathrm{~b}^{+} \mathrm{Ly} 6 \mathrm{G}^{-} \mathrm{SSC}^{\mathrm{hi}}\right)$ as described previously ${ }^{29}$.

\section{Histology and immunohistochemistry}

Lungs were perfused and fixed with $4 \%$ paraformaldehyde (Sigma-Aldrich) for $24 \mathrm{~h}$ at $4{ }^{\circ} \mathrm{C}$ and embedded in paraffin. Sections $2 \mu \mathrm{m}$ thick were stained with hematoxylin and eosin (H\&E), as described previously ${ }^{28}$. Immunohistochemistry was performed as previously described $^{28}$, using antibodies against Ki67 (Cell Signaling, Beverly, MA, USA; \#12202), Nkx2.1/TTF-1 (Dako/Agilent Technologies, Santa Clara, CA, USA; \#M3575) and Hmga2 (BioCheck, Foster City, CA, USA; \#59170AP). H\&E stained lung sections were imaged at low power and abnormal regions were masked on Image J software (Softonic International, Barcelona, Spain). Abnormal regions were classified as hyperplastic or adenoma/adenocarcinoma (ADC) based on morphology, and lesion number per lung was quantified. Hmga2 and Ki67 stained images were scored automatically using a custom Image $\mathrm{J}^{30}$ pipeline which utilized the Stardist plugin for nuclear segmentation $^{31}$. Nuclei were then scored based on their intensity in the brown channel after color deconvolution. Tumor burden was determined using Image J through a 
combination of rank and morphology filters, followed by an auto-threshold to select the whole tissue, and the regions of dense nuclei comprising the tumor.

\section{Cell growth analysis}

Cell lines were seeded from a single cell suspension derived from the left lobe of the lung. At each passage, cells were counted and $2 \times 10^{5}$ cells were re-seeded into one well of a 6-well plate in primary cell medium (DMEM/F-12+GlutaMAX (Thermo Fisher Scientific), 10\% FBS (Sigma-Aldrich), $100 \mathrm{U} / \mathrm{mL}$ penicillin (Thermo Fisher Scientific), $100 \mu \mathrm{g} / \mathrm{mL}$ streptomycin (Thermo Fisher Scientific), $0.04 \mathrm{mg} / \mathrm{mL}$ hydrocortisone (SigmaAldrich), $1 \times$ Insulin-Transferrin-Selenium-Ethanolamine (ITS-X; Thermo Fisher Scientific) and $5 \mathrm{ng} / \mathrm{mL}$ epidermal growth factor (Sigma-Aldrich)).

\section{Statistical analysis}

Prism (Version 8; GraphPad) software was used for all statistical analyses. Two-group comparisons were made using two-tailed Student's $t$-tests assuming equal variances and multivariate comparisons were performed using one-way ANOVA (Kruskal-Wallis test) with Tukey's multiple comparisons. Animal survival data were plotted using Kaplan-Meier curves and compared using log-rank (Mantel-Cox) test.

\section{Results and discussion}

\section{Loss of Zmat3 does not accelerate Myc driven lymphoma} development

To interrogate the role of Zmat3 in tumorigenesis in vivo, we investigated the collaboration of loss of Zmat 3 with deregulated expression of the oncogene $c-M y c$, using the $E \mu-M y c$ model of pre-B/B lymphoma development ${ }^{24}$. Critically, $E \mu-M y c$ mice have been extensively used to study the defects in the p53-pathway (e.g. impact of loss of Puma or loss of one allele of $p 53$ ) on tumorigenesis ${ }^{22,24,32}$. First, we examined the expression of Zmat3 in pre-leukemic $E \mu-M y c$ pre-B lymphoid cells isolated from the bone marrow of 4-week old $E \mu-M y c$ mice, with or without activation of p53. Indeed, Zmat 3 mRNA expression was induced in preleukemic pre-B lymphoid cells in response to both the MDM2 inhibitor Nutlin3a ${ }^{33}$ and $\gamma$-irradiation (Fig. 1a), confirming that Zmat3 is a bona fide p53 target gene. It has been shown that loss of one allele of $p 53$ significantly accelerates lymphoma development in $E \mu-M y c$ mice $^{3,11,24}$. Therefore, we next investigated whether loss of Zmat3 would accelerate lymphoma development in $E \mu-M y c$ mice. To address this question, Zmat3 knockout $\left(Z_{m a t}{ }^{-1-}\right)$ mice $^{14}$ were crossed with $E \mu-M y c$ transgenic mice ${ }^{24}$ and monitored for lymphoma development. Strikingly, neither loss of one allele or even both alleles of Zmat 3 altered the onset of lymphoma in $E \mu-M y c$ mice (Fig. 1b). Post-mortem analysis demonstrated no significant difference in leukemic burden, the overall proportion of pre-B $v s$ mature B-cell tumors, or in the weight of hematopoietic organs between sick $E \mu-M y c ; Z_{m a t}{ }^{-1-}$ vs E $\mu-M y c$ mice (Fig. 1c, d). Taken together, these results reveal that, unlike loss of $p 53$, germline loss of Zmat3 fails to accelerate c-MYC-driven lymphoma development.

\section{The impact of Zmat3 loss on adult lung development}

To further investigate the role of Zmat3 in oncogene driven tumorigenesis, we chose a lung adenocarcinoma (LUAD) model, in which p53 has been shown to play a critical tumor suppressive role ${ }^{34}$. In this model, activated $\mathrm{Kras}^{\mathrm{G} 12 \mathrm{D}}$ drives the development of lung adenomas, which progress to adenocarcinomas in the absence of $\mathrm{p} 53^{34}$. First, we examined the expression of Zmat 3 in lungs isolated from wt and $\mathrm{Zmat}^{-1-}$ mice, with or without activation of p53 by $\gamma$-irradiation. Indeed, Zmat3 mRNA expression was markedly induced in wt lung tissue in response to DNA damage, but absent in the lung tissues from $\mathrm{Zmat}^{-/-}$mice (Supplementary Fig. 1a). To determine whether the loss of Zmat3 impacted normal development and homeostasis of the lung, we performed an in-depth examination of the cellular composition and immune microenvironment of the lung tissue from $Z$ mat 3 deficient mice. As $Z m a t 3^{-1-}$ mice are viable and healthy ${ }^{14}$, we aged $Z m a t 3^{-1-}$ and Zmat $^{+/+}$(wt) littermate controls to adulthood (16 to 18 weeks old) and evaluated the histopathology and cellularity (epithelial and immune) of the lungs of these mice. The lungs of $\mathrm{Zmat}^{-1-}$ mice were histologically indistinguishable from the lungs of $p 53^{-1-}$ or wt control animals (Fig. 2a). Moreover, no differences in lung cellularity or weight were observed between $\mathrm{Zmat}^{-1-}$ and controls (Supplementary Fig. 1b, c). Furthermore, the immune cell composition of the lungs was unchanged, with the levels of resident myeloid and lymphoid cell populations comparable between $\mathrm{Zmat}^{-/-}, \mathrm{p} 53^{-/-}$and wt mice (Fig. 2b). To further investigate the role of Zmat3 in lung epithelium we generated primary cell cultures from $\mathrm{Zmat}^{-1-}, \mathrm{p} 53^{-/-}$and wt adult lungs and examined the impact of the loss of Zmat3 on cell proliferation and cellular morphology in vitro. Cumulative cell counts (over 8 passages) revealed that $\mathrm{Zmat}^{-1-}$ lung epithelial cells, similar to those from wt mice but unlike $p 53^{-1-}$ epithelial cells, failed to bypass replicative senescence (Fig. 2c-e). Consequently, similar to wt control cells, Zmat3 $^{-1-}$ cells underwent growth arrest exhibiting flattened appearance and multi-nucleation, characteristic of cells undergoing senescence (Fig. 2e). Collectively, these results show that the loss of Zmat3 has no impact on the development, homeostasis or immune cell composition of the murine lung and does not allow lung epithelial cells to evade cellular senescence in culture. 


\section{a}
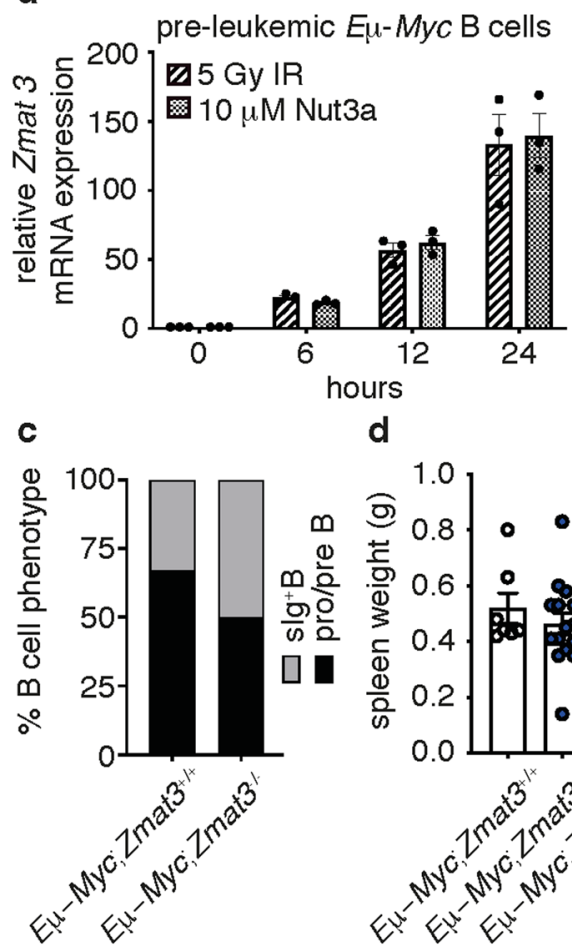

b
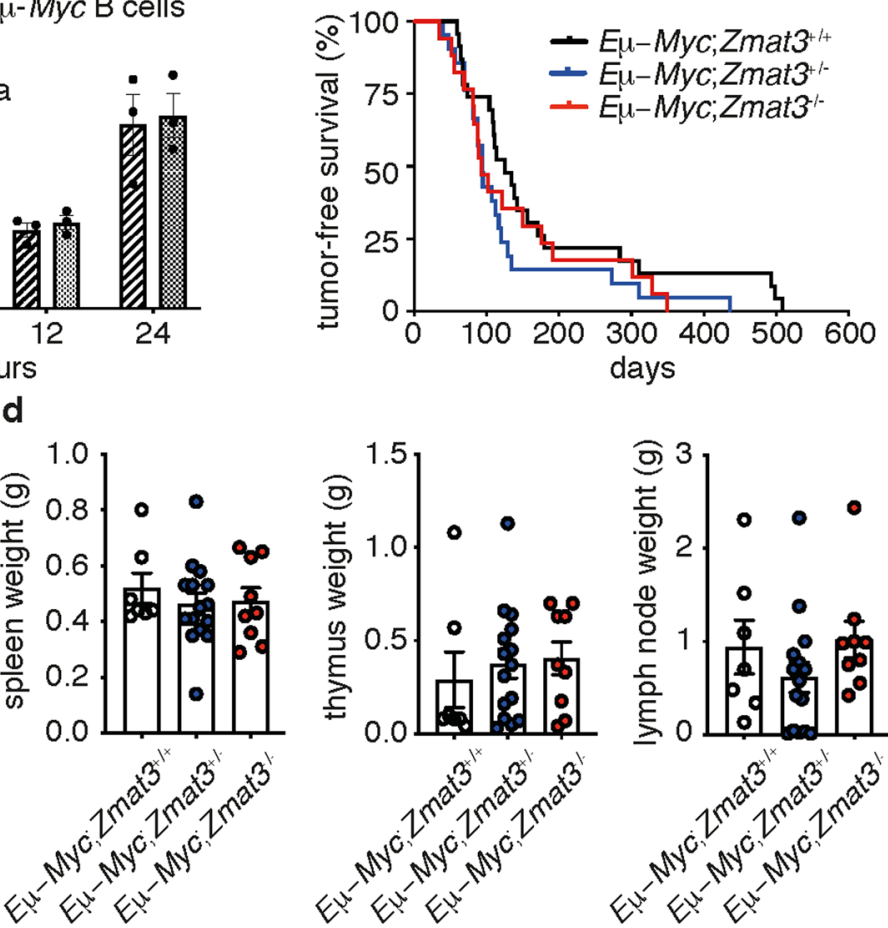

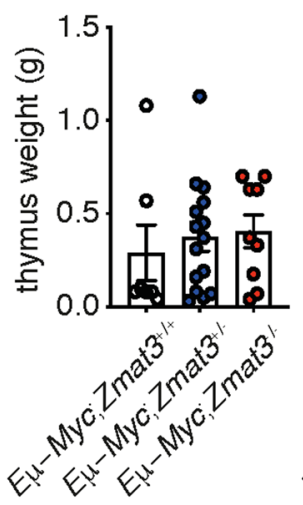

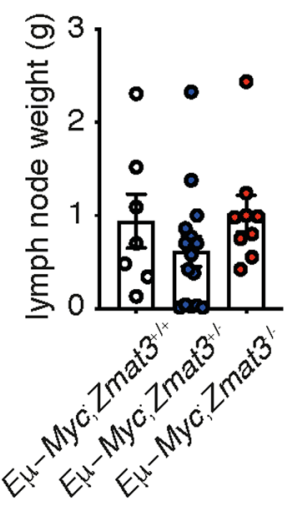

Fig. 1 Loss of Zmat3 has no impact on lymphoma development in Eu-Myc mice. a Zmat3 mRNA expression in pre-leukemic E $\mu$-Myc B lymphoid cells $0,6,12$ and $24 \mathrm{~h}$ following $5 \mathrm{~Gy}$-irradiation (IR) or treatment with $10 \mu \mathrm{M}$ Nutlin3a (Nut3a). Mean \pm SEM. $\mathbf{b}$ Lymphoma-free survival of E $\mu$-Myc

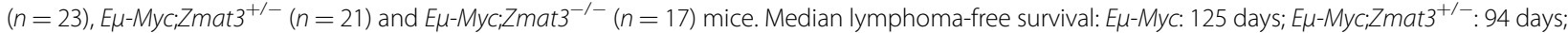

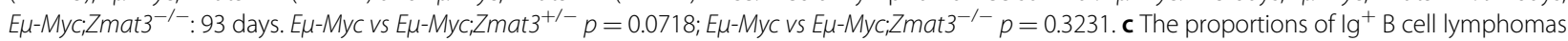
and pro-B/pre-B cell lymphomas in sick E $\mu-M y c ; Z m a t 3^{-1-}(n=4)$ and sick $E \mu-M y c(n=3)$ mice. No significant differences were observed; $p=0.5$

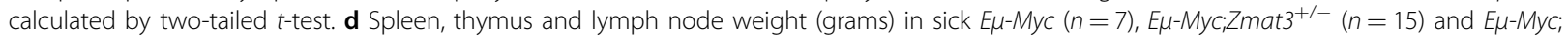
Zmat $^{-1-}(n=9)$ mice at ethical endpoint. Mean \pm SEM.

Germline loss of Zmat3 does not recapitulate $p 53-$ mediated acceleration of $\mathrm{Kras}^{\mathrm{G12D}}$-induced tumorigenesis

Next, to functionally evaluate the significance of Zmat3 in KRAS-mutant LUAD, we crossed $\mathrm{Zmat}^{-/-}$mice with $\mathrm{Kras}^{\mathrm{LSL}-\mathrm{G} 12 \mathrm{D} /+}$ mice $^{21}$. Cohorts of $\mathrm{Kras}^{\mathrm{LSL}-\mathrm{G} 12 \mathrm{D} /+}$ (hereafter; K), Kras ${ }^{\mathrm{LSL}-\mathrm{G} 12 \mathrm{D} /+} ; \mathrm{Zmat}^{-/-}$(hereafter; KZ) mice and Kras $^{\mathrm{LSL}-\mathrm{G} 12 \mathrm{D} /+} ; p 53^{-/-}$(hereafter; KPnull) mice were infected by intranasal inhalation with the ubiquitous Ad5-CMVCre adenovirus to recombine and activate mutant $\mathrm{Kras}^{\mathrm{G} 12 \mathrm{D}}$ expression in the respiratory epithelium ${ }^{27}$ (Fig. 3a). Consistent with previous reports utilizing a p53 conditional allele $^{21}$, germline loss of $p 53$ significantly accelerated lung cancer development in Kras $^{\mathrm{G} 12 \mathrm{D}}$ mice (median survival K: 151 days vs KPnull: 72 days; $p<0.0001$ ) (Fig. 3b). In contrast, loss of Zmat3 did not significantly alter the survival of Kras $^{\mathrm{G} 12 \mathrm{D}}$ mice (median survival: KZ: 138 days versus K: 151 days) (Fig. 3b). Consistent with the animal survival data, we observed no differences in tumor histology between the lungs from the $\mathrm{K}$ vs $\mathrm{KZ}$ mice, nor the proliferation index, as determined by Ki67 staining (Fig. 3c, Supplementary Fig. 2a). The tumors from both $\mathrm{K}$ and $\mathrm{KZ}$ mice were defined as highly differentiated, reflected by high Nkx2.1 expression and low to absent expression of Hmga2 (Fig. 3c). In contrast, tumors from KPnull mice displayed increased numbers of Ki67 and Hmga2 positive nuclei, indicative of more aggressive and more poorly differentiated adenocarcinoma lesions (Fig. 3c, Supplementary Fig. 2b), consistent with the crucial tumor suppressive function of p53 in mutant KRASdriven LUAD.

To evaluate whether Zmat3 loss impacts tumor initiation following oncogenic Kras ${ }^{G 12 D}$ activation, we performed a time course study, whereby $\mathrm{K}, \mathrm{KZ}$ and KPnull mice were analyzed 6, 10 and 16 weeks following Ad5-CMV-Cre infection. While hyperplasic and adenomatous lesions were only apparent in lungs of KPnull mice 6 weeks following Ad5-CMV-Cre infection (Fig. 4a), sporadic lung lesions were observed in both $\mathrm{K}$ and $\mathrm{KZ}$ mice by 10 weeks, with further progression seen only in the lungs of KPnull mice (Fig. 4a). Quantification of the histopathology of lesions in KZ, KPnull and $\mathrm{K}$ mice 10 weeks following Ad5-CMV-Cre administration failed to reveal significant differences in tumor burden between $\mathrm{K}$ and $\mathrm{KZ}$ mice suggesting that Zmat3 loss does not 
a

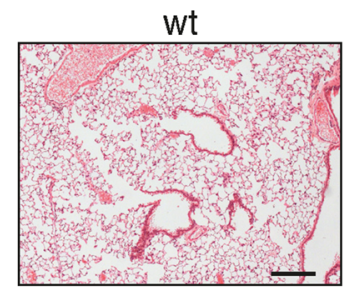

b

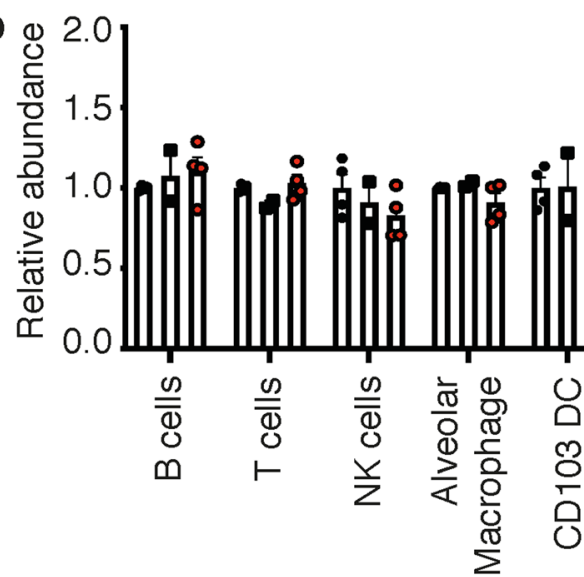

C

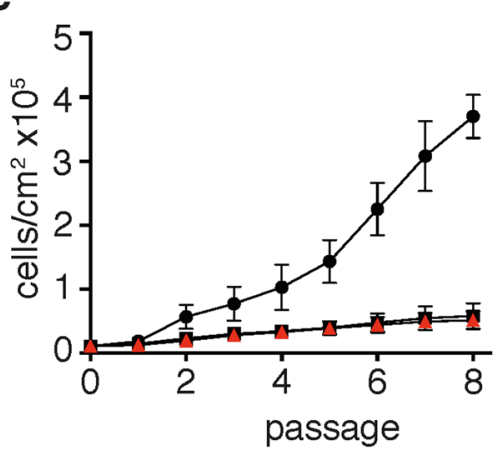

e

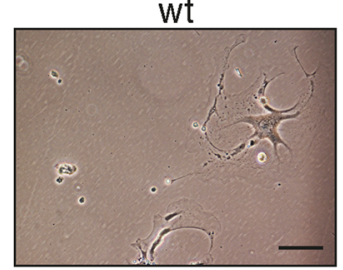

$\rightarrow-w t$

$p 53^{/-}$ $p 53^{\prime}$
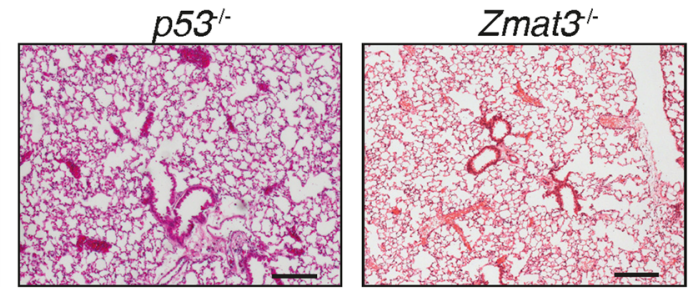

- wt

- $p 53^{-1-}$

- Zmat3-

$\rightarrow p 53^{--}$

- Zmat3 $^{\prime-}$

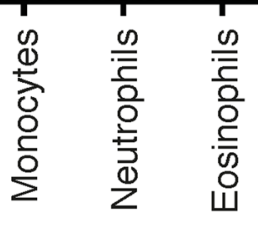

d
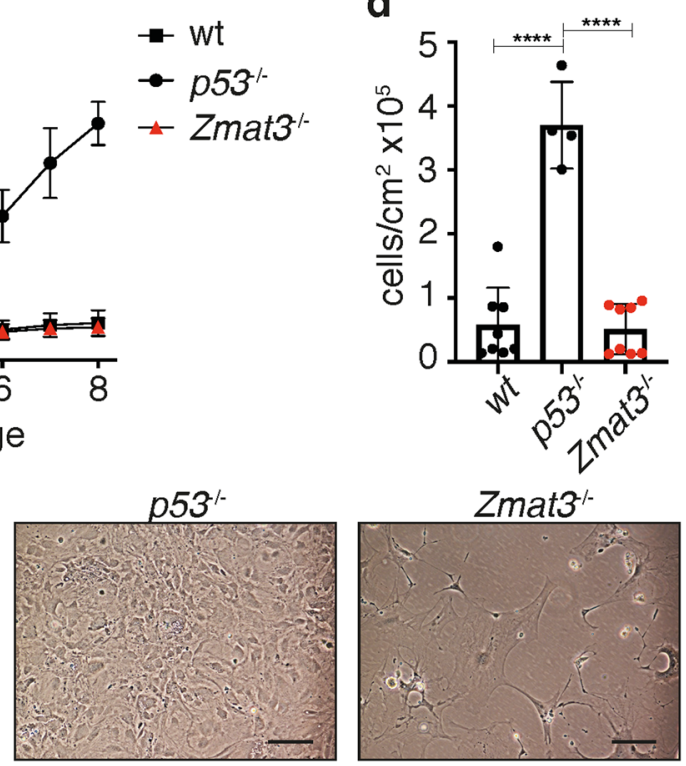

Fig. 2 No effect of Zmat3 loss in the adult lung. a Representative hematoxylin and eosin (H\&E) staining of lungs from $\mathrm{Zmat}^{-1-}$, littermate $\mathrm{Zmat}^{+/+}(\mathrm{wt})$ and $p 53^{-/-}$control mice aged 16-18 weeks. Scale, $200 \mu \mathrm{m}$. b Flow cytometric analysis of the lymphoid and myeloid cells present in the lungs from $\mathrm{Zmat}^{-1-}(n=4), p 53^{-1-}(n=2)$ and wt $(n=4)$ mice. Immune cell populations are normalized to wt control mice. $\mathbf{c}$ Growth analysis of primary cell lines derived from the lung epithelium of wt $(n=8), \mathrm{Zmat}^{-1-}(n=8)$ and $p 53^{-1-}(n=4)$ mice. Mean \pm SEM. $\mathbf{d}$ Accumulative cell number following 8 passages of primary wt $(n=8), p_{53^{-1-}}(n=4)$ and $Z$ mat3 $3^{-1-}(n=8)$ cell lines. Mean $\pm S D,{ }^{* * *} p<0.0001$. e Representative light microscope images of $\mathrm{Zmat}^{+/+}(\mathrm{wt}), \mathrm{p}^{2} 3^{-/-}$and $\mathrm{Zmat}^{-/-}$cell lines 8 passages following their derivation. Scale, $10 \mu \mathrm{m}$.

accelerate Kras $^{\mathrm{G} 12 \mathrm{D}}$-induced tumorigenesis (Fig. 4b, Supplementary Fig. 2c). These findings were further verified in mice analyzed 16 weeks following Ad5-CMV-Cre infection, with no difference in tumor burden detected between $\mathrm{K}$ and $\mathrm{KZ}$ mice (Supplementary Fig. 2d).
Next, we assessed the tumor immune microenvironment using multiparametric flow cytometry. Consistent with

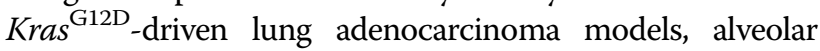
macrophages were abundant in the microenvironment of all genetic backgrounds tested, suggesting no alterations in this 


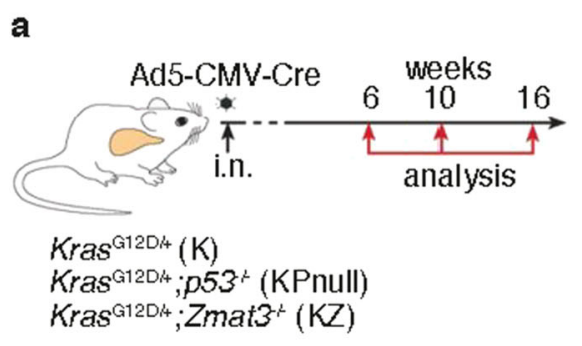

c
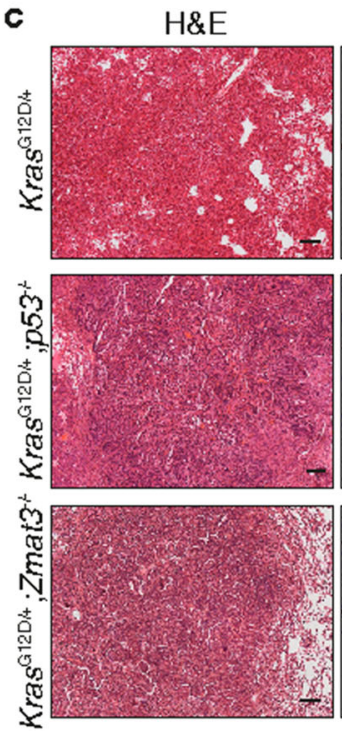

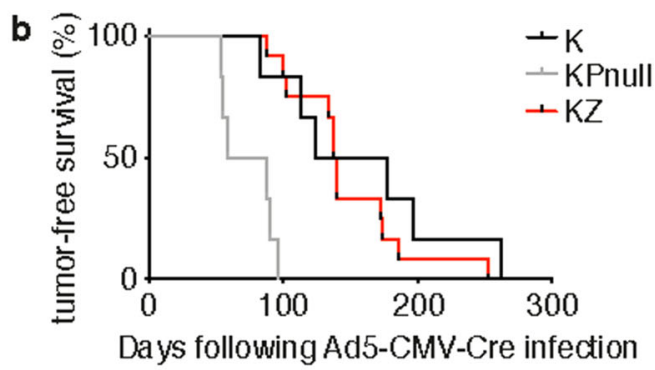

NkX2.1
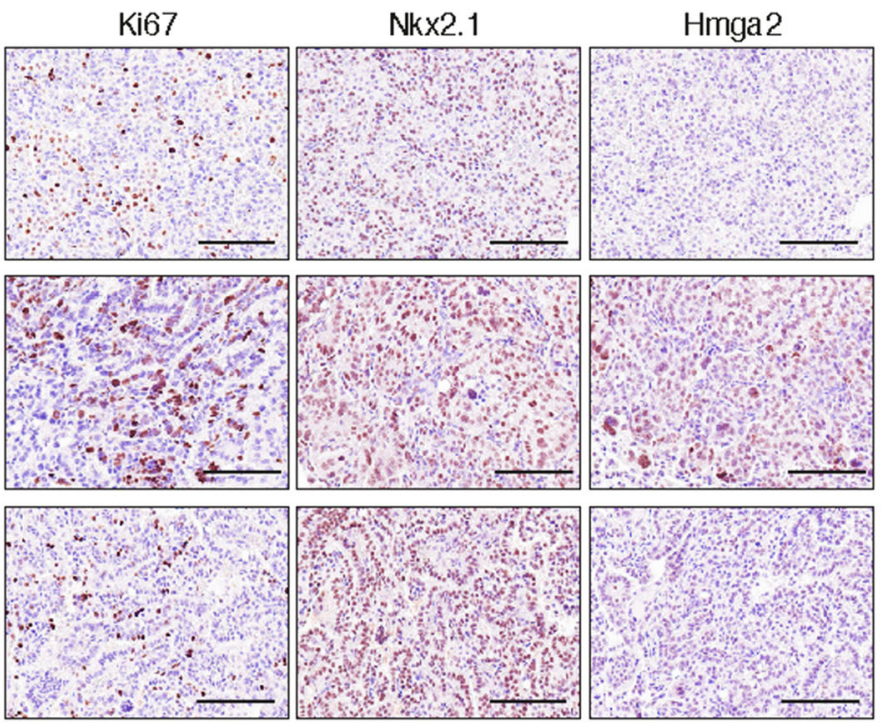

Fig. 3 Loss of Zmat3 does not collaborate with mutant Kras ${ }^{\mathrm{G} 12 \mathrm{D}}$ in the development of lung adenocarcinoma. a Schematic of experimental design. Kras ${ }^{\mathrm{LSL}-\mathrm{G} 12 \mathrm{D} /+}(\mathrm{K})$ mice $^{21}$ were crossed with $p 53^{-/-}$mice $^{37}$ (KPnull) or Zmat3 ${ }^{-/-}$mice ${ }^{14}$ (KZ). b Kaplan-Meier tumor-free survival analysis of K $(n=6), \mathrm{KPnull}(n=6)$ and $\mathrm{KZ}(n=12)$ mice following i.n. infection of Ad5-CMV-Cre virus. c Representative H\&E staining and immunohistochemistry of

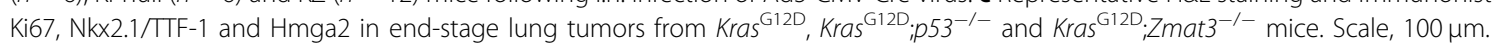

key feature of mutant ras $^{\mathrm{G} 12 \mathrm{D}}$-driven adenocarcinoma development due to the loss of $Z m a t 3^{35}$. Furthermore, no differences were identified in the lymphoid or myeloid compartments between the $\mathrm{K}, \mathrm{KZ}$ and KPnull cohorts (Supplementary Fig. 3a). Consistent with the presence of tumors in the lungs 10-weeks post Ad5-CMV-Cre administration, $\mathrm{CD}^{+} \mathrm{T}$ cells in the lungs of tumor-bearing mice were activated and displayed elevated PD-1 expression (Supplementary Fig. 3b). These findings show that loss of Zmat3 does not alter the key factors shown to impact the immune milieu under homeostatic conditions or in response to oncogene activation.

We have previously shown that lung weight is a robust readout of tumor burden in $\mathrm{Kras}^{\mathrm{G} 12 \mathrm{D}}$-driven GEMMs ${ }^{35}$. Interestingly, while lung weights were consistently in a relatively narrow range within the $\mathrm{K}$ (uniformly low lung weight) and KPnull cohorts (uniformly high lung weights), large variations in lung weights were observed between individual KZ mice (Fig. 4c). This may indicate some tumor suppressor function of Zmat3, perhaps dependent on oncogenic lesions cooperating with mutant $\mathrm{Kras}^{\mathrm{G} 12 \mathrm{D}}$ and the loss of Zmat3 that are present in some but not all $\mathrm{KZ}$ mice.

\section{Zmat3 loss fails to rescue $K \mathrm{Kas}^{\mathrm{G}}{ }^{2 \mathrm{D}}$ lung adenocarcinoma cells from oncogene-induced senescence}

Loss of p53 function prevents cell cycle arrest and enables evasion from cellular senescence in mutant KRAS-driven tumorigenesis. To assess whether loss of Zmat3 could promote evasion from oncogene-induced senescence (OIS) in $\mathrm{Kras}^{\mathrm{G} 12 \mathrm{D}}$ mutant lung epithelial cells, primary cell lines generated from $\mathrm{KZ}$ and KPnull lung tumors were assessed for proliferation over 8 passages. Consistent with previous findings ${ }^{9}$, cumulative cell counts demonstrated that while KPnull cells replicated indefinitely, and $\mathrm{KZ}$ cells underwent growth arrest that was evident after 4 passages (Fig. $5 \mathrm{a}-\mathrm{c}$ ). These results suggest that the loss of Zmat3, unlike the loss of $p 53$, does not permit evasion from OIS in $\operatorname{Kras}^{\mathrm{G} 12 \mathrm{D}}$ driven lung tumorigenesis. 


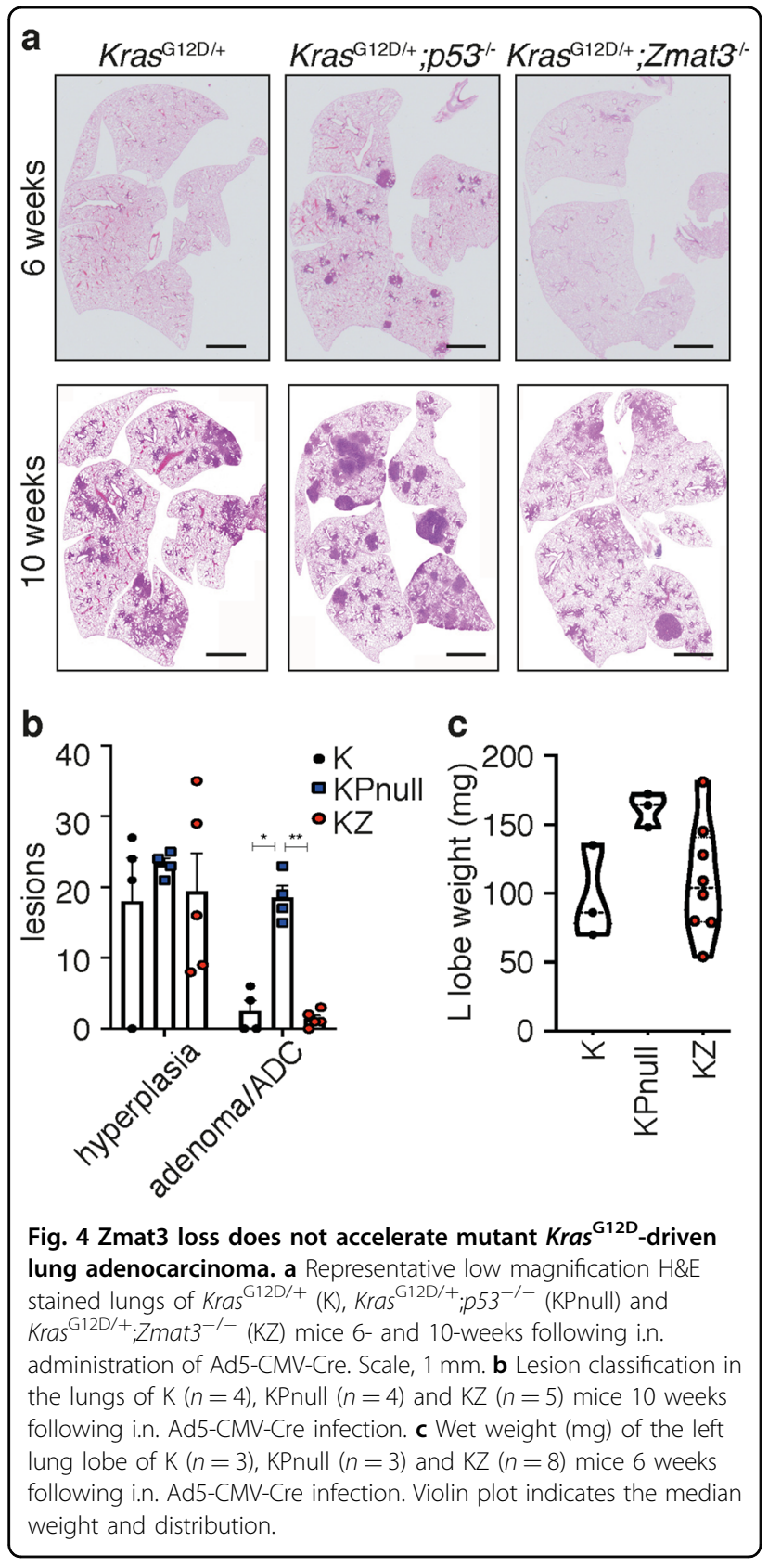

Taken together, we have demonstrated that in murine models of $M y c$-driven lymphoma and $K r a s^{\mathrm{G} 12 \mathrm{D}}$-driven LUAD, the germline loss of Zmat3 did not have a considerable effect on the rate of onset, overall incidence or severity of tumor development, unlike p53 loss. In humans, it has been shown that Zmat3 is expressed ubiquitously in all tissues and its deregulation has been implicated in various cancers, including leukemia as well as lung and breast cancer ${ }^{15-17}$. More importantly, our previous findings have shown that loss of Zmat3 causes leukemia only in overlap with concomitant loss of the p53 targets, Puma and $p 21^{14}$, that are critical for p53- mediated apoptosis and cell cycle arrest, respectively. Hence, it is possible that the tumor suppressive function of Zmat3 is more pronounced in other tissues and oncogene induced models. The understanding of the molecular mechanisms underlying p53s' function in tumor suppression is constantly expanding. It is now clear that for p53-mediated tumor suppression other functions in the cell are critical in addition to induction of cell cycle arrest, apoptosis or senescence ${ }^{4-9}$. Zmat3 is a p53 target gene that is involved in activation of cell cycle arrest or apoptosis indirectly, and as it regulates gene expression in a post-translational manner could modulate the p53 response towards other still unknown biological effector processes $^{15,36}$. The inability of loss of Zmat 3 to cooperate with $c-M Y C$ and $K R A S$ expression in tumorigenesis may be explained by the finding that Zmat3 regulates a large set of targets at the mRNA level ${ }^{36}$ and that its' function is dependent on additional co-occurring mutations other than deregulated $c-M Y C$ expression, mutations in KRAS and cellular stress (Fig. 5d). Further studies will be required to investigate the function and biological significance of Zmat3 in epithelial and lymphoid tumorigenesis.

\begin{abstract}
Acknowledgements
We are grateful to C. Gatt, K. McKenzie, S. Oliver and G. Siciliano for expert animal care; B. Helbert, K. Mackwell, and R. Chan for genotyping; E. Tsui in the WEHI Histology Facility and S. Monard in the WEHI Flow Cytometry Facility for expert support; E. Michalak for providing animal data and discussions. This work was supported by the Australian Phenomics Network (APN); the Australian National Health and Medical Research Council (NHMRC) Project Grant to M.J.H. (1145728), to M.J.H. and A.S. (1143105), Program Grant to A.S. (1016701); the Leukemia and Lymphoma Society of America to A.S. and M.J.H. (LLS SCOR 7001-13); the Cancer Council of Victoria Project grant to A.S. (1052309) and Venture Grant to M.J.H. and A.S.; support to A.S. from the estate of Anthony (Toni) Redstone OAM and a Spanish Ministry of Economy and Development Grant to A.J. (RTI2018-099017-A-I00). K.D.S. is supported by a Victorian Cancer Agency Mid-Career Research Fellowship (18003) and the Peter and Julie Alston Centenary Fellowship, A.J. is supported by Ramon y Cajal Fellowship (RYC2018-025244-I), A.S. and M.J.H. are supported by NHMRC Fellowships (1020363 and 1156095), M.S.B is supported by Cancer Council Victoria Postdoctoral Fellowship. This work was made possible through the Victorian Government Operational Infrastructure Support and Australian Government, a "Maria de Maeztu" Programme for Units of Excellence in R\&D MDM-2014-0370 (Government of Spain) and La Caixa banking foundation (51110009).
\end{abstract}

\section{Author details}

${ }^{1}$ The Walter and Eliza Hall Institute of Medical Research, 1G Royal Parade, Parkville, Melbourne, VIC 3052, Australia. '2Department of Medical Biology, University of Melbourne, Parkville, Melbourne, VIC 3052, Australia. ${ }^{3}$ Department of Experimental and Health Sciences, Universitat Pompeu Fabra, Doctor Aiguader 88, 08003 Barcelona, Spain

\section{Conflict of interest}

The authors declare that they have no conflict of interest.

\section{Publisher's note}

Springer Nature remains neutral with regard to jurisdictional claims in published maps and institutional affiliations. 

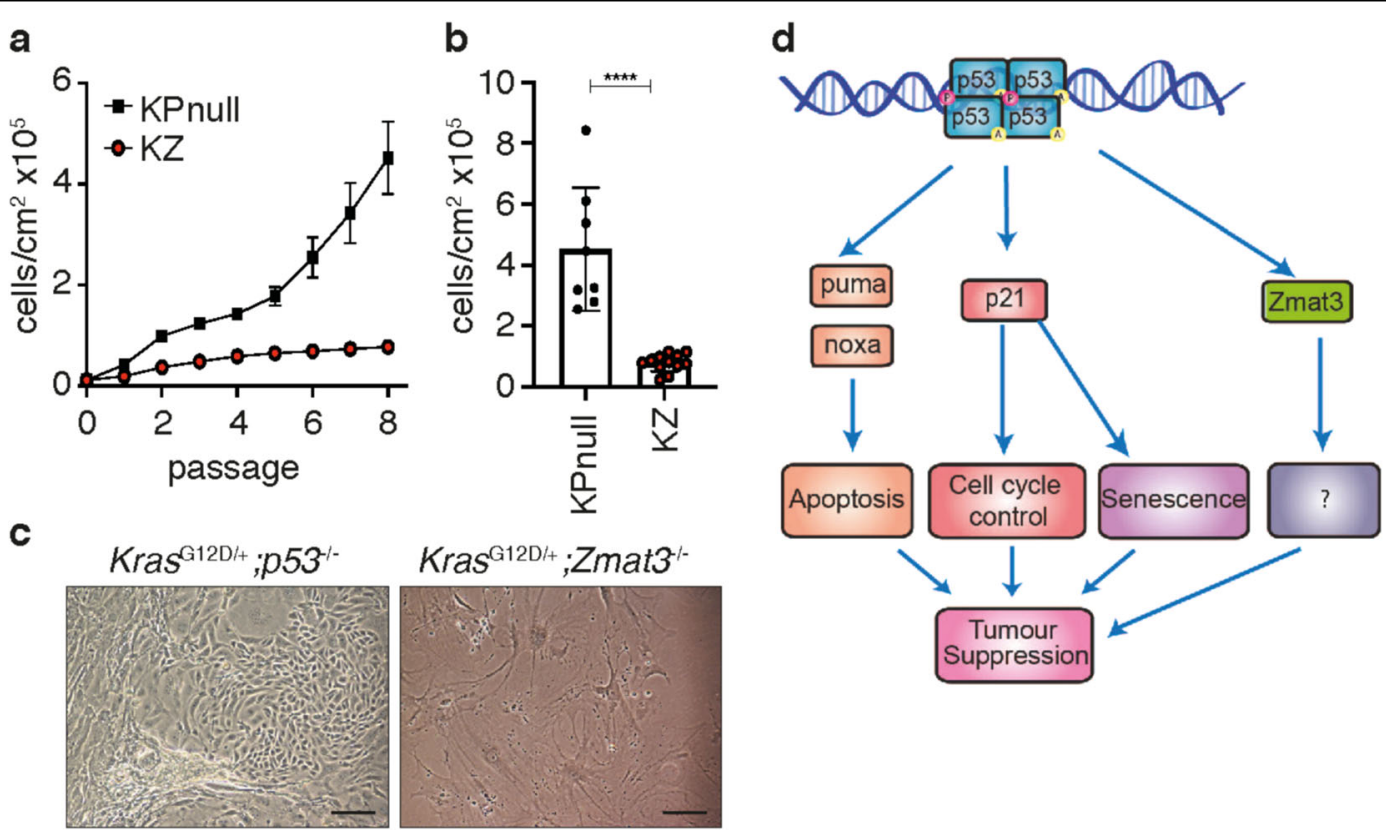

Fig. 5 Zmat3 loss does not overcome mutant Kras $^{\mathbf{G 1 2 D}}$ induced cell senescence. a Growth analysis of primary cell lines derived from the lung epithelium of KPnull $(n=8)$ and KZ $(n=12)$ mice 10 weeks following i.n. Ad5-CMV-Cre infection. Mean \pm SEM. $\mathbf{b}$ Accumulative cell number following 8 passages of primary KPnull $(n=8)$ and KZ $(n=12)$ cell lines. Mean $\pm S D$, ${ }^{* * *} p<0.0001$. c Representative light microscope images of Kras ${ }^{G 12 D} / p 53^{-1-}$ (KPnull) and $\mathrm{Kras}^{\mathrm{G} 12 \mathrm{D}} / \mathrm{Zmat}^{-/-}$(KZ) cell lines 8 passages following their derivation. Scale, $10 \mu \mathrm{m}$. $\mathbf{d}$ Schematic representation of p53 target genes and their role in tumor suppression.

Supplementary Information accompanies this paper at (https://doi.org/ 10.1038/s41419-020-03066-9).

Received: 6 February 2020 Revised: 10 July 2020 Accepted: 13 July 2020 Published online: 20 October 2020

\section{References}

1. Attardi, L. D. \& Jacks, T. The role of p53 in tumour suppression: lessons from mouse models. Cell. Mol. Life Sci. 55, 48-63 (1999).

2. Bieging, K. T., Mello, S. S. \& Attardi, L. D. Unravelling mechanisms of p53mediated tumour suppression. Nat. Rev. Cancer 14, 359-370 (2014).

3. Aubrey, B. J., Kelly, G. L., Janic, A., Herold, M. J. \& Strasser, A. How does p53 induce apoptosis and how does this relate to p53-mediated tumour suppression? Cell Death Differ. 25, 104-113 (2018).

4. Kastenhuber, E. R. \& Lowe, S. W. Putting p53 in context. Cell 170, 1062-1078 (2017).

5. Mello, S. S. \& Attardi, L. D. Deciphering p53 signaling in tumor suppression. Curr. Opin. Cell Biol. 51, 65-72 (2018).

6. Brady, C. A. \& Attardi, L. D. p53 at a glance. J. Cell Sci. 123, 2527-2532 (2010).

7. Valente, L. J. et al. p53 Efficiently suppresses tumor development in the complete absence of its cell-cycle inhibitory and proapoptotic effectors p21, puma, and noxa. Cell Rep. 3, 1339-1345 (2013).

8. Brady, C. A. et al. Distinct p53 transcriptional programs dictate acute DNAdamage responses and tumor suppression. Cell 145, 571-583 (2011).

9. Li, T. et al. Tumor suppression in the absence of p53-mediated cell-cycle arrest, apoptosis, and senescence. Cell 149, 1269-1283 (2012).

10. Mello, S. S. et al. A p53 super-tumor suppressor reveals a tumor suppressive p53-Ptpn14-Yap axis in pancreatic cancer. Cancer Cell $\mathbf{3 2}$, 460-473.e6 (2017).

11. Valente, L. J., Grabow, S., Vandenberg, C. J., Strasser, A. \& Janic, A. Combined loss of PUMA and p21 accelerates C-MYC-driven lymphoma development considerably less than loss of one allele of p53. Oncogene 35, 3866-3871 (2016).

12. Li, T. et al. Tumor suppression in the absence of p53-mediated cell cycle arrest, apoptosis, senescence. Cell 149, 1269-1283 (2012).

13. Brady, C. A. et al. Distinct p53 transcriptional programs dictate acute DNAdamage responses and tumor suppression. Cell 145, 571-583 (2011).

14. Janic, A. et al. DNA repair processes are critical mediators of p53-dependent tumor suppression. Nat. Med. 24, 947-953 (2018).

15. Vilborg, A., Bersani, C., Wilhelm, M. T. \& Wiman, K. G. The p53 target Wig-1: a regulator of mRNA stability and stem cell fate? Cell Death Differ. 18, 1434-1440 (2011).

16. Bersani, C., Xu, L.-D., Vilborg, A., Lui, W.-O. \& Wiman, K. G. Wig-1 regulates cell cycle arrest and cell death through the p53 targets FAS and 14-3-30. Oncogene 33, 4407-4417 (2014).

17. Hellborg, F. et al. Human wig-1, a p53 target gene that encodes a growth inhibitory zinc finger protein. Oncogene 20, 5466-5474 (2001).

18. Vilborg, A. et al. The p53 target Wig-1 regulates $p 53$ mRNA stability through an AU-rich element. Proc. Natl Acad. Sci. USA 106, 15756-15761 (2009).

19. Vilborg, A., Wilhelm, M. T. \& Wiman, K. G. Regulation of tumor suppressor p53 at the RNA level. J. Mol. Med. 88, 645-652 (2010).

20. Kim, B. C. et al. Wig1 prevents cellular senescence by regulating p21 mRNA decay through control of RISC recruitment. EMBO J. 31, 4289-4303 (2012).

21. Jackson, E. L. et al. The differential effects of mutant p53 alleles on advanced murine lung cancer. Cancer Res. 65, 10280-10288 (2005).

22. Garrison, S. P. et al. Selection against PUMA gene expression in Myc-driven Bcell lymphomagenesis. Mol. Cell. Biol. 28, 5391-5402 (2008).

23. Michalak, E. M. et al. Puma and to a lesser extent Noxa are suppressors of Mycinduced lymphomagenesis. Cell Death Differ. 16, 684-696 (2009).

24. Adams, J. M. et al. The c-myc oncogene driven by immunoglobulin enhancers induces lymphoid malignancy in transgenic mice. Nature 318, 533-538 (1985).

25. Donehower, L. A. et al. Mice deficient for p53 are developmentally normal but susceptible to spontaneous tumours. Nature 356, 215-221 (1992).

26. Jacks, T. et al. Tumor spectrum analysis in p53-mutant mice. Curr. Biol. 4, 1-7 (1994). 
27. DuPage, M., Dooley, A. L. \& Jacks, T. Conditional mouse lung cancer models using adenoviral or lentiviral delivery of Cre recombinase. Nat. Protoc. 4 1064-1072 (2009).

28. Best, S. A., Kersbergen, A., Asselin-Labat, M. L. \& Sutherland, K. D. Combining cell type-restricted adenoviral targeting with immunostaining and flow cytometry to identify cells-of-origin of lung cancer. In Methods in Molecular Biology 15-29 (Humana Press Inc., 2018).

29. Best, S. A. et al. Synergy between the KEAP1/NRF2 and PI3K pathways drives non-small-cell lung cancer with an altered immune microenvironment. Cell Metab. 27, 935-943.e4 (2018)

30. Schindelin, J. et al. Fiji: An open-source platform for biological-image analysis. Nat. Methods 9, 676-682 (2012).

31. Schmidt, U., Weigert, M., Broaddus, C. \& Myers, G. Cell detection with starconvex polygons. In Medical Image Computing and Computer Assisted Intervention - MICCAI 2018. 265-273 (2018).
32. Michalak, E. M. et al. Puma and to a lesser extent Noxa are suppressors of Myc-induced lymphomagenesis. Cell Death Differ. 16, 684-696 (2009).

33. Vassilev, L. T. et al. In vivo activation of the p53 pathway by smallmolecule antagonists of MDM2. Science 303, 844-848 (2004).

34. Jackson, E. L. et al. The differential effects of mutant p53 alleles on advanced murine lung cancer. Cancer Res. 65, 10280-10288 (2005)

35. Best, S. A. et al. Distinct initiating events underpin the immune and metabolic heterogeneity of KRAS-mutant lung adenocarcinoma. Nat. Commun. 10, 4190 (2019).

36. Bersani, C. et al. Genome-wide identification of Wig-1 mRNA targets by RIPSeq analysis. Oncotarget 7, 1895-1911 (2016).

37. Jacks, T. et al. Tumor spectrum analysis in p53-mutant mice. Curr. Biol. 4, 1-7 (1994). 